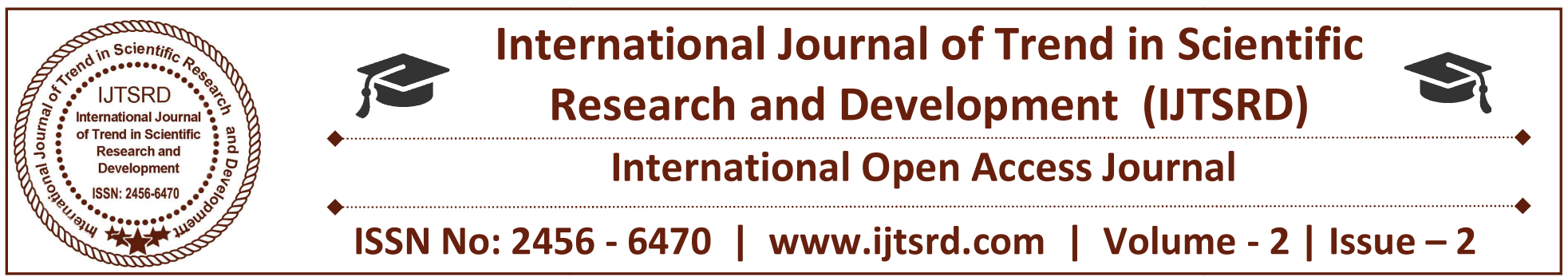

\title{
Biosynthesis and Characterization of Zinc Oxide Nanoparticles using Onion Bulb Extract
}

\author{
N. Tensingh Baliah \\ Post Graduate and Research Department \\ of Botany, Ayya Nadar Janaki Ammal \\ College, Sivakasi, Tamil Nadu, India
}

\author{
S. Lega Priyatharsini \\ Post Graduate and Research Department \\ of Botany, Ayya Nadar Janaki Ammal \\ College, Sivakasi, Tamil Nadu, India
}

\section{ABSTRACT}

The wide application of nanoparticles stimulates the need for synthesizing them but, the conventional methods are usually hazardous and energy consuming. This leads to focus on "green synthesis" of nanoparticles which seems to be easy efficient and ecofriendly approach. In this study, the plant mediated synthesis of zinc oxide nanoparticles ( $\mathrm{ZnO}$ NPs) was carried out using bulb extract of Allium cepa as a reducing agent. The optimized nano zinc thus obtained was quantified and characterized using UVVisible spectroscopy, Fourier Transform Infrared Spectroscopy (FT-IR), X-Ray diffraction, Scanning Electron Microscope (SEM), EDAX and Zeta potential analyses. Further, the synthesized ZnO NPs were tested for antimicrobial activity.

Keywords: Nanoparticles, zinc oxide, biosynthesis, Allium cepa

\section{INTRODUCTION}

Nanotechnology is a emerging field with its application in science and technology for the purpose of manufacturing new materials at nano scale level (Albrecht et al., 2006). Recent advance in the field of nanotechnology, particularly the ability to prepare highly ordered nanoparticles of any size and shape (Sangeetha et al., 2012). Nanotechnology is a multidisciplinary scientific field undergoing explosive development. The nanometer-sized particles offer novel structural, optical and electronic properties that are not attainable with individual molecules or bulk solids (Anitha et al., 2011). The characters of metal and metal oxide nanoparticles have been of great interest due to their distinctive feature such as catalytic activity, optical, magnetic and electrical properties (Garima et al, 2010). Nanoparticles interaction with biological materials and established a series of nanoparticle / biological interfaces that depend on colloidal forces as well as dynamic biophysicochemical interactions. These interactions lead to the formation of new nanomaterial with control size shape, surface chemistry, roughness and surface coatings.

The use of plants for the synthesis of nanoparticles is novel and provides a cost-effective and environmentally friendly alternative to chemical and physical synthesis. In addition, the use of plants can be easily scaled up for large-scale synthesis without the use of toxic chemicals or the need for high pressures, energy and temperature (Bhainsa et al., 2017). Plant-mediated biosynthesis of zinc oxide nanoparticles has been achieved in Aloe barbadensis (Sangeetha et al., 2011), Physalis alkekengi (Qu et al., 2011), Parthenium hysterophorus (Rajiv et al., 2013), Zingiber officinale (Bhuyan et al., 2015), Azadirachta indica (Wang et al., 2014), Ocimum basilicum, Medicago sativa (Nagaiyothi et al., 2013), Anisochilus carnosus (Bala et al., 2015), milky latex of Calotropis procera and fruit juice of Citrus aurantifolia (Samat et al., 2013) etc. 
It is known that the plant mediated synthesis of $\mathrm{ZnO}$ nanoparticles is much safer and environmentally friendly as compared to chemical synthesis. The size of the synthesized $\mathrm{ZnO}$ nanoparticles was in the range of $60-70 \mathrm{~nm}$. The larger nanoparticles of $\mathrm{ZnO}$ resulted from the agglomeration of smaller nanoparticles. Highly ionic nanoparticulate metal oxides such as zinc oxide nanoparticles are unique in that they can be produced with high surface areas and with unusual crystal structures (Anandraj et al., 2017). Compared to organic materials, inorganic materials such as $\mathrm{ZnO}$ possess superior durability, greater selectivity and heat resistance (Padmavathy and Vijayaraghavan, 2008).

\section{MATERIAL AND METHODS}

\section{SYNTHESIS NANOPARTICLES \\ ZINC \\ OXIDE}

The onion bulbs were washed with sterile distilled water and the outer covering of the bulb was manually peeled off and the fleshy part of the onion was rewashed with sterile distilled water. The onion bulb was cut into small pieces and $10 \mathrm{~g}$ of bulb was ground using mortar and pestle with distilled water. The extraction was filtered using muslin cloth and then Whatmann No.1 filter paper and used as reducing agent and stabilizer. Zinc nitrate was used as precursor for the synthesis of zinc oxide nanoparticles. For $\mathrm{ZnO}$ NPs synthesis, the reaction mixture was prepared by mixing of $10 \mathrm{ml}$ of leaf extract and $90 \mathrm{ml}$ of $1 \mathrm{mM}$ zinc nitrate solution in a $250 \mathrm{ml}$ Erlenmeyer conical flask. This mixture was incubated in dark conditions at $37^{\circ} \mathrm{C}$. Then, the mixture was centrifuged at $10,000 \mathrm{rpm}$ for 20 minutes. The pellet was taken after centrifugation and air dried. The pellet was used for further studies.

\section{RECOVERY OF ZnO NANOPARTICLES}

The nanoparticles thus obtained were purified by repeated centrifugation at $10000 \mathrm{rpm}$ at $25^{\circ} \mathrm{C}$ for 10 minutes. It was followed by re-dispersion of the pellet in deionized water to get rid of any uncoordinated biological molecules. The process of centrifugation and re-dispersion were repeated with sterile distilled water to ensure better separation of free entities from the nanoparticles.

\section{CHARACTERIZATION \\ OF NANOPARTICLES}

The synthesized nanoparticles were characterized by UV-Visible spectroscopy, Scanning Electron Microscopy (SEM), Energy Dispersive X-ray Spectroscopy Analysis (EDAX), Fourier Transform Infrared Spectroscopy (FTIR), X-Ray Diffraction (XRD) and Zeta potential analyses.

\section{UV-VISIBLE SPECTROSCOPY ANALYSIS}

The reduction of pure metal ions was confirmed by measuring the absorption of reaction mixture by UVVis Spectrophotometer (UV 1700 SHIMADZU) from 200 to $800 \mathrm{~nm}$ (Roy et al., 2013).

\section{FOURIER TRANSFORM INFRARED (FTIR)} ANALYSIS

FTIR has become an important tool for understanding the involvement of functional groups in interactions between metal particles and biomolecules. FT-IR spectra were recorded at $1 \mathrm{~cm}^{-1}$ resolution by FTIR spectrophotometer (FTIR-8400S SHIMADZU) using $\mathrm{KBr}$ pellet technique (Prathra et al., 2011). The frequency range was measured as wave numbers typically over the range $4000-400 \mathrm{~cm}^{-1}$.

\section{X-RAY DIFFRACTION (XRD) ANALYSIS}

To determine the nature and size of the $\mathrm{ZnO}$ nanoparticles, X-ray diffraction (XRD) was performed. The pellet was dissolved in deionized sterile water and washed thrice in the same by centrifugation. The pellet was retained and air dried. The powder from of the sample was coated on the XRD grid, the spectra were recorded $40 \mathrm{kV}$ and a current of $30 \mathrm{~mA}$ with $\mathrm{CuK} \alpha$ radiation using XRD (Philips PW1050/37 model). The diffracted intensities were recorded from $20^{\circ} \mathrm{C}$ to $80^{\circ}$ at $2 \theta$ angles. The crystalline nature of synthesized nanoparticles was calculated from the width of the XRD peaks, using the Debye-Scherrer formula $(D=K \lambda / \beta \cos \theta)$.

\section{SCANNING ELECTRON MICROSCOPY (SEM) ANALYSIS}

The synthesized nanoparticles were dispersed in water and the resultant suspensions were homogenized using ultra sonicator for one to two hours. A drop of the nanoparticles suspension was placed on a piece of micro glass slide attached to a metal grid coated with carbon film and dried it gradually at room 
temperature. The sample was then sputter coated with gold and visualized with a JEOL JSM-6480 LV SEM to assess the particle size, shape and percentage (Forough and Farhadi, 2010).

\section{ENERGY DISPERSIVE SPECTROSCOPY (EDAX) ANALYSIS}

A drop of the nanoparticles suspension was placed on a piece of microglass slide attached to a metal grid coated with carbon film, and dried it gradually at room temperature. X-ray spectrometer (EDAX) operated at an accelerating voltage at $10 \mathrm{KeV}$. The sample was then sputter coated with gold and visualized with a BRUKER to assess the particle size, shape and percentage of synthesized particles.

\section{ZETA POTENTIAL ANALYSIS}

The supernatant was filtered and then sonicated for 5 minutes. The solution was centrifuged for 15 minutes at $25^{\circ} \mathrm{C}$ with $5000 \mathrm{rpm}$ and the supernatant was collected. Then, the supernatant was diluted for 4 to 5 times and then set for Zeta-Potential analysis (Mohammed et al., 2014).

\section{ANTIMICROBIAL ACTIVITY}

The $\mathrm{ZnO}$ nanoparticles were tested against the bacterial pathogens such as E.coli (ATCC25922), Staphylococcus aureus (ATCC9144), Pseudomonas aeruginosa (ATCC25619) and fungal pathogens Aspergillus niger (MTCC 1344) and Aspergillus flavus (MTCC 1973). Agar disc diffusion method (Baur et al., 2012) was employed for the study of antimicrobial activity of the synthesized zinc oxide nanoparticles.

\section{STATISTICAL ANALYSIS}

The XRD values were inferred through JCPDS file no 89-3722. Further, the UV-Visible spectrum, FTIR, XRD values were interpreted by using ORIGIN VERSION-8 (data analysis and graphing work space). The data were reported as mean $\pm \mathrm{SE}$ and in the figure parentheses represent the per cent activity.

\section{RESULTS \& DISCUSSION}

\section{VISUAL OBSERVATION OF ZINC OXIDE NANOPARTICLES}

The synthesized zinc oxide nanoparticles were initially confirmed by visual observation by colour change in the reaction medium. The metal ions were reduced during the exposure to aqueous extract of onion within 24 hours of incubation period. It was observed that the colour of the reaction medium was changed to pale yellow to pale brown colour (Fig. 1).

\section{UV-VISIBLE SPECTROSCOPY ANALYSIS}

The reduction of zinc oxide ions in the onion extract was further confirmed by UV-Vis Spectrophotometer. $\mathrm{UV}-\mathrm{Vis}$ absorption spectra of nanoparticles were shown in (Fig. 2). The UV-Vis absorption spectrum of $\mathrm{ZnO}$ NPs was as the peak maxima in $354 \mathrm{~nm}$ which corresponding to the absorbance of zinc oxide nanoparticles. After incubation period, the colour was changed because of the excitation of Surface Plasmon Vibrations in the $\mathrm{ZnO}$ nanoparticle. The reduction of zinc was subjected to analysis by using the UV-Vis Spectrophotometer. Absorption spectra of ZnO NPs formed in the reaction media has absorbance peak at $310 \mathrm{~nm}$, broadening of peak indicated that the particles are polydispersed. The frequency and width of the surface plasmon absorption depends on the size and shape of the metal nanoparticles as well as on the dielectric constant of the metal itself and the surrounding medium (Melvin et al., 2009).

The aqueous extract of Punica granatum and zinc nitrate solution, the colour of the reaction medium changed rapidly from colour less to brownish yellow. The colour indicated surface plasmon vibration typical of zinc oxide ( $\mathrm{ZnO}$ ) nanoparticles. The maximum intensity at $364 \mathrm{~nm}$ was observed indicating complete reduction of zinc ions. The UV-Vis optical absorption spectrum of $\mathrm{ZnO}$ nanoparticles showed a sharp absorbance at $345 \mathrm{~nm}$, which indicated an almost uniform size of the nanoparticles. However, upon change in particle size or particle shape, a slight shift in the absorption was observed (Guo et al., 2001). The UV-Vis absorption spectrum of $\mathrm{ZnO}$ nanoparticles using flower extract of Nyctanthes arbor-tristis showed a sharp absorbance at $369 \mathrm{~nm}$ (Jamdagni et al., 2016).

\section{FOURIER TRANSFORM INFRARED SPECTROSCOPY (FTIR) ANALYSIS}

FTIR analysis was carried out to identify the biomolecules which were responsible for the reduction of metal ions into $\mathrm{ZnO}$ nanoparticles in the presence of onion extract (Fig. 3). The phytochemical found in the onion extract were responsible for the formation of various nanoparticles. The FT-IR spectrum of onion extract showed several absorption 
peaks ranged from $3421 \mathrm{~cm}^{-1}$ to $677 \mathrm{~cm}^{-1}$. The region of band was phenols, alkanes due to N-H stretching of proteins and $\mathrm{O}-\mathrm{H}$ stretching, $>\mathrm{C}=\mathrm{O}$ stretching of esters, aromatics, ring $\mathrm{C}-\mathrm{C}$ stretching of phenyl, alkanes, C-O stretch in vibration combined with the ring stretch of phenyl, aliphatic amines, alcohols, carboxylic acids, ester, ether, functional groups mainly from carbohydrate and alkyl halides. FTIR measurements of zinc oxide nanoparticles with onion extract showed the presence of bands at 3438.84, $2925.81,1633.59,1383.83,1122.49,1104.17$ and $600.78 \mathrm{~cm}^{-1}$. These bands were indicting the presence of N-H bend primary amines, C-O stretching alcohols, carboxylic acids, ester and ethers.

FTIR spectrum of $\mathrm{ZnO}$ nanoparticles revealed that the peak at $417.52 \mathrm{~cm}^{-1}$ was the characteristic absorption of $\mathrm{ZnO}$ bond and the broad absorption peak at $3438 \mathrm{~cm}^{-1}$ attributed to the characteristic absorption of hydroxyl group (Khan et al., 2011). The FTIR spectrums of $\mathrm{ZnO}$ NPs derived from Corymbia citriodora leaf extract showed the strong absorption peaks observed at 3300 and $1620 \mathrm{~cm}^{-1}$ and were assigned to $\mathrm{O}-\mathrm{H}$ stretch and $\mathrm{N}-\mathrm{H}$ bend functional groups. Weaker bands observed at 2955, 777 and $633 \mathrm{~cm}^{-1}$ were assigned to $-\mathrm{C}-\mathrm{H}$ stretch (alkanes), $\mathrm{C}-$ $\mathrm{H}$ (aromatics) and $-\mathrm{C}=\mathrm{C}-\mathrm{H}$ (alkynes). The absorption peaks at 1520 and $1431 \mathrm{~cm}^{-1}$ were attributed the $\mathrm{C}=\mathrm{C}$ bending and $\mathrm{C}-\mathrm{C}$ stretching of aromatic rings, respectively. The peak at $1053 \mathrm{~cm}^{-1}$ assigned to the $\mathrm{C}-\mathrm{N}$ stretching mode in aliphatic amines. The formation of $\mathrm{ZnO}$ NPs may attribute to the chemicals presence in the $C$. citriodora such as citronellal, linalool, catechin, gallic acid, courmaric acid and protocatechuric acid, which acted as reducing agents as well as stabilizing agents (Tamuly et al., 2013).

The FTIR spectrum of $\mathrm{ZnO}$ nanoparticles derived from Ocimum tenuiflorum showed the fundamental mode of vibration at $3458.04 \mathrm{~cm}^{-1}$ which corresponded to the $\mathrm{O}-\mathrm{H}$ stretching vibration, $1625.35 \mathrm{~cm}^{-1}$ which correspond to the N-H bend, $1418.86 \mathrm{~cm}^{-1}$ which corresponded to C-C stretching vibration of alcohol, carboxlic acid, ether and ester were confirmed. The band at $1148.10 \mathrm{~cm}^{-1}$ corresponds to $\mathrm{C}-\mathrm{N}$ symmetric stretching vibration and $\mathrm{O}-\mathrm{H}$ bending of the hydroxyl group at $3458.04 \mathrm{~cm}^{-1}$ was observed. The absorption at $875.23 \mathrm{~cm}^{-1}$ was due to the formation of tetrahedral coordination of $\mathrm{Zn}$. The bond at $835.61 \mathrm{~cm}^{-1}$ was due to the $\mathrm{C}-\mathrm{Cl}$ stretching vibration. The peak at $668.29 \mathrm{~cm}^{-1}$ indicated the stretching vibrations of $\mathrm{ZnO}$ nano particle which was consistent with that reported before (Ravichandrika et al., 2012). From FTIR analysis of leaves extract of Ocimum tenuiflorum all functional groups play important role in the preparation of zinc oxide nanoparticles.

Green synthesis of $\mathrm{ZnO}$ nanoparticles using Phyllanthus embilica stem extract revealed The FT-IR spectra showed the presence of bonds due to $\mathrm{O}-\mathrm{H}$ stretching around $3423 \mathrm{~cm}^{-1}$. Peak at $1405 \mathrm{~cm}^{-1}$ may be assigned to symmetric stretching of the carbonyl side groups in the amino acid residues of the protein molecules. The band at $1022 \mathrm{~cm}^{-1}$ was corresponding to $\mathrm{C}-\mathrm{N}$ stretching vibration of amine. The peak at around $1340 \mathrm{~cm}^{-1}$ present in green $\mathrm{ZnO}$ signified amide III band of the random coil of protein (Joel and Sheik, 2017).

\section{X-RAY DIFFRACTION PATTERN ANALYSIS}

The various peaks in the XRD pattern could be assigned to the crystalline zinc oxide phase with the hexagonal wurzite structure with the lattice parameters $\mathrm{a}=3.2475 \mathrm{~nm}$ and $\mathrm{c}=3.2501 \mathrm{~nm}$. The XRD pattern showed different intensity peaks in the whole spectrum of $2 \theta$ values ranging from $20^{\circ}$ to $80^{\circ}$ for the onion. The Allium cepa extract-mediated synthesized $\mathrm{ZnO}$ nanoparticles were indexed as (100), (002), (101), (102), (110), (103), (200), (112) and (201). The zinc was indexed as (100) and (101). The average crystalline size was determined using Scherrer's equation. The average crystalline size of zinc oxide nanoparticles was 20-100 nm (Fig. 4). Zinc oxide nanoparticles synthesized using Corymbia citriodora leaf extract showed the peaks at $32.1^{\circ}, 34.6^{\circ}, 36.1^{\circ}$, $47.7^{\circ}, 56.4^{\circ}, 63.1^{\circ}$ and $68.1^{\circ}$ and indexed to hexagonal wurtzite $\mathrm{ZnO}$ (JCPDS 36-1451). Further, no other peaks have been detected in the sample and confirmed the biosynthesis of ZnO NPs owning a high purity. The mean crystalline size was found to be 21 nm (Yuhong Zheng et al., 2017). Biological synthesis of zinc oxide nanoparticles from Catharanthus roseus leaf extract showed strong diffraction peaks at 20,32, 35 and 40 degrees of $2 \mathrm{q}$ which corresponds to 111 , 200, 220 and 311 crystal planes, which were significant agreement with the JCPDS file $36145(\mathrm{a}=$ $\mathrm{b}=3.249 \mathrm{~A}^{0}, \mathrm{C}=5.206 \mathrm{~A}^{0}$ ) and indexed as the hexagonal wurtezite structure of $\mathrm{ZnO}$. High purity and crystallinity of the synthesized $\mathrm{ZnO}$ NPs were also confirmed. 


\section{SCANNING ELECTRON MICROSCOPE (SEM) ANALYSIS}

The surface morphology of the nanoparticles was characterized using Scanning Electron Microscopy. The onion extract mediated $\mathrm{ZnO}$ nanoparticles were found as nano-rod shape (Fig. 5). The biological approach using milky latex of Calotropis procera has been used for the first time as a reducing material as well as surface stabilizing agent for the synthesis of spherical-shaped ZnO NPs. The structure, phase and morphology of synthesized product were investigated by the SEM analysis (Sangeetha et al., 2011). Raut et al. (2017) done the SEM analysis for zinc oxide nanoparticles synthesized by Ocimum tenuiflorum leaf extract. The SEM analysis showed the hexagonal nanoparticle formed with diameter range $11-25 \mathrm{~nm}$. $\mathrm{ZnO}$ nanoparticles synthesized using Trifolium pratense flower extract revealed that SEM images of the $\mathrm{ZnO}$ nanoparticles were agglomerated with a particle size ranging from 100-190nm (Dobrucka and Dugaszewska, 2015).

\section{EDAX ANALYSIS}

EDAX analysis is very much useful for further confirmation of presence of nanoparticles. The EDAX analysis showed the confirmative peaks for $\mathrm{ZnO}$ NPs. The $\mathrm{ZnO}$ nanoparticles were confirmed by typical absorption peak at $1 \mathrm{KeV}$ (Fig. 6). Sharma et al. (2014) reported that the size of the nanoparticle is more than that calculated from the Debye -Scherrer formula indicating the agglomeration of crystallites in $\mathrm{ZnO}$ nanoparticles. The aggregation of particles should have been originated from the large specific surface area and high surface energy of $\mathrm{ZnO}$ nanoparticles. The aggregation occurred probably during the process of drying (Raoufi, 2013). The green synthesis of zinc oxide nanoparticles using Moringa oleifera leaf extract the strong and weak peaks are observed from $\mathrm{Zn}$ and $\mathrm{O}$ atom and weak peaks are observed form S, K, C, P, Ca element (Mishra et al., 2015). Biosynthesis and characterization of $\mathrm{ZnO}$ nanoparticles using the aqueous leaf extract of Imperata cylindrica the EDS spectrum shows the high value of zinc $(80.3 \%)$ and oxygen $(19.65 \%)$.

\section{ZETA POTENTIAL ANALYSIS}

Zeta potential measures the potential stability of the nanoparticles in the colloidal suspension. From the Zeta potential analysis, onion extract mediated $\mathrm{ZnO}$ nanoparticles carried a charge of $\pm 26.5 \mathrm{mV}$ (Fig. 7).
The zeta potential of the biologically synthesized NPs was determined in water as dispersant. The zeta potential was found to be -20 to $-30 \mathrm{mV}$ for $\mathrm{ZnO}$ nanoparticles. The high negative value approved the repulsion among the particles and thereby increases in stability of the structure of nanoparticles (Jafarirad et al., 2016). It was suggested that the surface of the nanoparticles was negatively charged and dispersed in the medium. The negative value confirms the repulsion among the particles and proves that they are very stable (Anandalakshmi et al., 2016). The biosynthesis of zinc oxide nanoparticles using Ixora coccinea leaf extract zeta potential was found to be $-49.19 \mathrm{mV}$ (Yedurkar et al., 2016). The synthesis and characterisation of zinc oxide nanoparticles using terpenoid fractions of Andrographis paniculata leaves the zeta potential of synthesized $\mathrm{ZnO}$ nanoparticles was $17.6 \mathrm{mV}$.

\section{ANTIMICROBIAL ACTIVITY}

Antimicrobial activity of onion extract mediated zinc oxide nanoparticles were tested against bacterial pathogens such as E.coli and Staphylococcus aureus, Pseudomonas aeruginosa and two fungal pathogens like Aspergillus niger and Aspergillus flavus. The antibacterial activity (inhibition zone) of zinc oxide nanoparticles was higher against Pseudomonas aeruginosa followed by Staphylococcus aureus and E.coli. In the case of fungal pathogens, the maximum inhibition zone was observed against $A$. niger (Table $1)$.

Nanoparticles possess the antimicrobial properties against several microorganisms including bacteria, fungi and certain extremophiles (Haeed et al., 2008). The properties of $\mathrm{ZnO}$ nanoparticle is strongly influenced by the particle size and mechanism of cell inhibition. The properties include disruption of cell membrane, altering the permeability, electrostatically binding to the cell surface and accumulation of nanoparticle in cytoplasm (Stoimenov et al., 2002). Antimicrobial activity of zinc oxide nanoparticles were known from the very distant past and has many applications in disinfecting medical devices, water purification, and wound healing, creams, lotions and antibacterial creams. The mechanism of action of antimicrobial activity zinc oxide is similar to other nanoparticles, but it acts mostly through destruction of bacterial walls. Zinc oxide nanoparticles have been widely used against Gram-positive and Gram negative bacteria (Emami et al., 2013). 
The mechanisms of antibacterial activity of $\mathrm{ZnO}$ NPs were direct contact of $\mathrm{ZnO}$ NPs with cell walls, resulting in destructing bacterial cell integrity, liberation of antimicrobial ions mainly $\mathrm{Zn}^{+2}$ ions. However, the toxicity mechanism varies in various media as the species of dissolved $\mathrm{Zn}$ may change according to the medium components besides the physicochemical properties of ZnO NPs (Yedurkar et al., 2016). Nano-sized $\mathrm{ZnO}$ exhibited varying morphologies and showed a significant antibacterial activity over a wide spectrum of bacterial species explored by a large body of researchers. $\mathrm{ZnO}$ is currently being investigated as an antibacterial agent in both microscale and nanoscale formulations. $\mathrm{ZnO}$ exhibited significant antimicrobial activities when particle size is reduced to the nanometer range, then nano-sized $\mathrm{ZnO}$ interacted with bacterial surface and/or with the bacterial core where it entered inside the cell and subsequently exhibited distinct bactericidal mechanisms (Seil and Webster, 2012).

\section{CONCLUSIONS}

The biological production of metal nanoparticles is becoming a very important field in chemistry, biology and materials science. Metal nanoparticles have been produced chemically and physically for a long time; however, their biological production has only been investigated very recently. The biological reduction of metals by plant extracts has been known since the early 1900s; however, the reduction products were not studied. The rapid biological synthesis of zinc nanoparticles using bulb extract of Allium cepa provides an environmental friendly, simple and efficient route for synthesis of nanoparticles. The use of plant extracts avoids the usage of harmful and toxic reducing and stabilizing agents. The synthesized nano particles of $\mathrm{ZnO}$ are in the range of 20-100 nm. Zinc nanoparticles can exist in ions only in the presence of strong oxidizing substances. The synthesis of $\mathrm{ZnO}$ nano particles is still in its infancy and more research needs to be focused on the mechanism of nanoparticle formation which may lead to fine tuning of the process ultimately leading to the synthesis of nanoparticles with a strict control over the size and shape parameters.

\section{ACKNOWLEDGEMENT}

The authors are thankful to the Management and the Principal of Ayya Nadar Janaki Ammal College, Sivakasi, Tamil Nadu, India for providing laboratory facilities to carry out the experiment.

\section{REFERENCES}

1. Albrecht, M. A., Evan, C. W. \& Raston, C. L. (2006). Green chemistry and the health implications of nanoparticles. Green Chemistry, 3(8), 417-432.

2. Anand Raj, L.F.A. \& Jayalakshmy, E. (2017). Biosynthesis and characterization of zinc oxide nanoparticles using root extract of Zingiber officinale. J.Chem, 31, 51-56.

3. Anandalakshmi, K., Venugobal, J. \& Ramasamy, V. (2016). Characterization of zinc oxide nanoparticles by green synthesis method using Pedalium murex leaf extract and their antibacterial activity. Appl. Nanosci. 6(2), 399.

4. Anitha, S. Kiruba, D., Singhal, G. (2011). Bhavesh, R., Kunalkasaraiya, M., AshishRajanan, S. \& Singh R. Biosythesis of zinc oxide nanoparticles using Ocimum sanctum (Tulsi) leaf extract and screening its antimicrobial activity, $J$. Nanopart. Res, 13(9), 2981-2988.

5. Bala, N., Saha, S., Chakraborty, M., Maiti, M., Das, S., Basub, R. \& Nandyc, P. (2015). Green synthesis of zinc oxide nanoparticles using Hibiscus subdariffa leaf extract: Effect of temperature on synthesis, anti-Bacterial activity and anti-diabetic activity. Int. J. Mol. Sci, 5, 49935003.

6. Baur, R.W., Kirby, M. D. K., Sherris, J. C. \& Turck, M. (2012). Antibiotic susceptibility testing by standard single disc diffusion method. Americ. J. Clin. Pathol. 45, 493-496.

7. Bhainsa K. C. \& Souza, D. (2017). Extracellular biosynthesis of zinc oxide nanoparticles using the fungus Aspergillus fumigates. Biosurfaces, 47(2), 160-164.

8. Bhuyan, T., Mishra, K., Khanuja, M. \& Prasad, R. (2015). Biosynthesis of zinc oxide nanoparticles from Azadirachta indica for antibacterial and photocatalytic applications. Mat. Sci. Semiconduct. Process, 32, 55-56.

9. Dobruckaand, R. \& Dugaszewska, J. (2015). Biosynthesis and antibacterial activity of $\mathrm{ZnO}$ nanoparticles using Trifolium pratense flower extract. Saudi J. Biol. Sci. 23, 517-523.

10. Emami, S., Akya, A., Hossain Zadeh, A. \& Barkhordar, S. (2013). Bacterial contamination of traditional ice creams in Kermanshah. Iran $J$. Med. Microbiol. 7(2), 59-62.

11. Forough, M. \& Farhadi, K. (2010). Biological and green synthesis of zinc oxide nanoparticles. Turkish J. Eng. Env. Sci. 34, 281 - 287. 
12. Garima, S., Bhavesh, R., KunalKasariya, M., AshishRanjan S. \& Singh, R. (2011). Biosynthesis of zinc oxide nanoparticles using Octimum sanctum (Tulsi) leaf extract and screeming its antimicrobial activity. J. Nanopart. Res, 13, 2981-2988.

13. Guo, L., Cheng, X. Y., Yan, Y. J. \& Ge, W. K. (2001). Synthesis and optical properties of crystalline polymer-capped $\mathrm{ZnO}$ nanorods. Mat. Sci. Engin, 16, 123-127.

14. Haeed, A., Hasan, M., Raffi, M., Hussain, F. \& Bhatti, T. M. (2008). Antibacterial characterization of zinc oxide nanoparticles against E.Coli ATCC-15224. J. Mater. Sci. Technol, 24(2), 192-196.

15. Jafarirad, S., Mehrabi, M., Divband, B. \& KosariNasab, M. (2016). Biofabrication of zinc oxide nanoparticles using fruit extract of Rosa canina and their toxic potential against bacteria: a mechanistic approach. Mater. Sci. Eng. 59, 296302.

16. Jamdagni , P., Poonam Khatri, J. \& Rana, S. (2016). Green synthesis of zinc oxide nanoparticles using flower extract of Nyctanthes arbor-tristis and their antifungal activity. J. King. Saud.Univers. Sci. 3(8), 417-432.

17. Joel, C. \&. Sheik M. B. (2017). Green synthesis of $\mathrm{ZnO}$ nanoparticles using Phyllanthus embilica stem extract and their antibacterial activity. Der. Pharmacia. Lettre. 8 (11), 218-223.

18. Khan, S. B. and Mohamed, R. (2011). Low temperature growth of $\mathrm{ZnO}$ nanoparticles, photocatalyst and acetone sensor. Talanto, 85, 943.

19. Melvin Joe, M., Jayochitra, J. \& Vijayapriaya, M. (2009). Antimicrobial activity of some common spices against certain human pathogens. J. Med. Plants Res, 3, 1134-1136.

20. Mishra, A., Mishra D. K. \& Bohra, N. K. (2015). Synthesis and characterization of zinc oxide nanoparticles by Azadirachta indica leaves. Annals Arid Zone 54(1\&2), 43-49.

21. Mohammed, J. Haider \& Mehdi, M. S. (2014). Study of morphology and zeta potential analyzer for the zinc oxide nanoparticles. Int. J. Scienti. Engineer. Res, 5(7), 381-387.

22. Nagajyothi, P. C., An, T. N. M., Sreekanth, T. V. M., Lee, D. J. \& Lee, K. D. (2013). Green route biosynthesis: characterization and catalytic activity of Zno nanoparticles. Mat. Lett, 108(7), 160-163.
23. Padmavathy, N. \& Vijayaraghavan R. (2008). Enhanced bioactivity of $\mathrm{ZnO}$ nanoparticles-an antimicrobial study. Sci. Technol. Adv. Mater. 9: 432-438.

24. Prathra, T. C. N., Chandrasekaran, A.M., Raichur, A. \& Mukherjee. (2011). Biomimetic synthesis of zinc oxide nanoparticles by Citrus limon (Lemon) aqueous extract and theoretical prediction of particle size. Colloid. Surf. B: Bioint. 82, 152-159.

25. Qu, J., Yuan, X., Wang, X. \& Shao, P. (2011). Zinc accumulation and synthesis of $\mathrm{ZnO}$ nanoparticles using Physalis alkekengi L. Environ. Pollut, 159, 1783-1788.

26. Rajiv, P., Rajeshwari, S. \& Venckatesh, R. (2013). Bio-fabrication of zinc oxide nanoparticles using leaf extract of Parthenium hysterophorus L. and Its size-dependent antifungal activity against plant fungal pathogens. Environ. Sci. Technol, 112, 384-387.

27. Raoufi, D. (2013). Synthesis and microstructural properties of $\mathrm{ZnO}$ nanoparticles prepared by the precipitation method. Renewable Energy, 50, 932-937.

28. Raut, S., Thorat, P.V. \& Thakre, R. (2017). Green synthesis of zinc oxide ( $\mathrm{ZnO})$ nanoparticles using Ocimum tenuiflorum leaves. Int. J. Sci. Res. 4 (5), 1225 - 1228.

29. Ravichandrika, P., Kiranmayi, and Ravikumar, R.V.S. S. N. 2012. Synthesis, characterization and antibacterial activity of $\mathrm{ZnO}$ nanoparticles. Int. J. Pharma. Pharm. Sci. 4(4), 336-338.

30. Roy, S., Triparna, M., shatarupa, T. \& Das, P. (2013). Biosynthesis, characterization and antifungal activity of zinc oxide nanoparticles synthesized by the fungus Aspergillus foetidus. J. Nanometer. Biostruc. 8, 197-205.

31. Samat, N.A. \& Nor, R.M. (2013) Sol-Gel synthesis of zinc oxide nanoparticles using Citrus Aurantifolia extracts. Int. J. Sci. Res. 39, 545-548.

32. Sangeetha, G., Rajeshwari, S. \& Venckatesh, R. (2011). Green synthesis of zinc oxide nanoparticles by Aloe barbadeneis Miller. leaf extract: Structure and optical properties. Mat. Res. Bull, 46, 2560-2566.

33. Sangeetha, G., Sivaraj, R. \& Venckatesh, R. (2012). Green synthesized $\mathrm{ZnO}$ nanoparticles against bacterial and fungal pathogens. Progress in Natural science: Materials International, 22(6), 693-700.

34. Seil, J. T. \& Webster, T. J. (2012). Antimicrobial applications of nanotechnology: methods and literature. Int. J. Nanomed, 7(2), 2767-2781. 
35. Sharama, G., Jasuja, N. D., Ali, M. I. \& Joshi, S. C. (2014). A review on nanomedicinal and nanosensing potential of nanoparticles. Int. J. Biol. Chem, 8 (2), 58-84.

36. Stoimenov P. K., Klinger R. L., Marchin G. L., \& Klabunde K. J. 2002. Metal oxide nanoparticles as bactericidal agents. Langmuir 18(17), 6679- 6686.

37. Tamuly, M., Hazarikaa, S., Borah, M. R., Das \& M. P. Boruah. (2013). In situ biosynthesis of Ag, $\mathrm{Au}$ and bimetallic nanoparticles using Piper pedicellatum: Green chemistry approach. Colloids Surf. B Biointerf. 102, 627-634.

38. Wang, X.X., Wu, L., Zhou, P., Li, C., Zhao, L.B., An. W. and Chen, Y. (2014) Effect of ZnO nanoparticles on Medicago sativa at the germination stage. Appl. Mechan. Mat. 665, 583586.

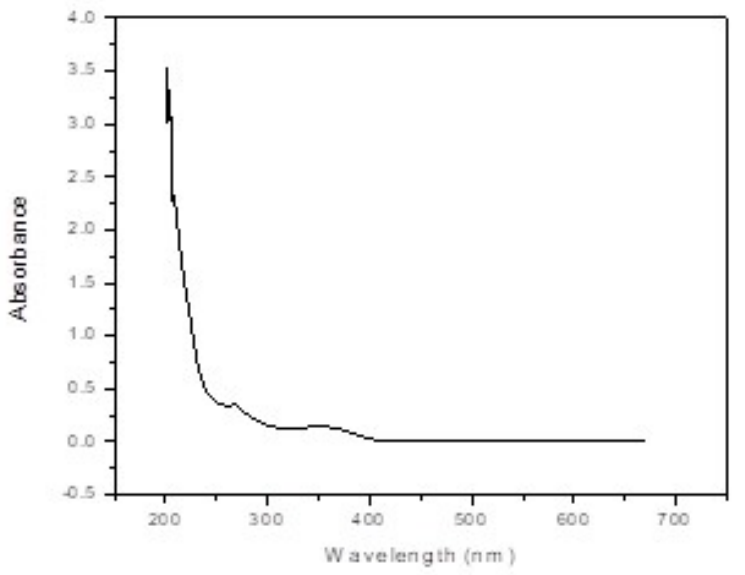

Fig. 2: UV-Visible spectrum of Zinc oxide nanoparticles

39. Yedurkar, S., Maurya, C. \& Mahanwar P. (2016). Biosynthesis of Zinc Oxide nanoparticles using Ixora coccinea leaf extract - a green approach. Open J. Syn. Theo. Appli.5, 1-14.

40. Yuhong Zheng, Li Fu, Fuigui Han, Aiwu Wang, Wen Cai, Jinping Yu, Jun Yang \& Feng Peng (2017). Green biosynthesis and characterization of zinc oxide nanoparticles using Corymbia citriodora leaf extract and their photocatalytic activity. Green Chem. Lett. Rev. 8(2), 59-63.

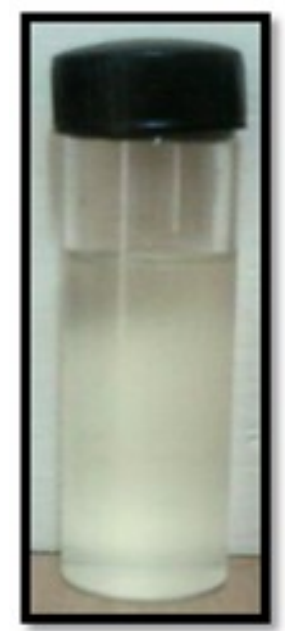

Onion Extract

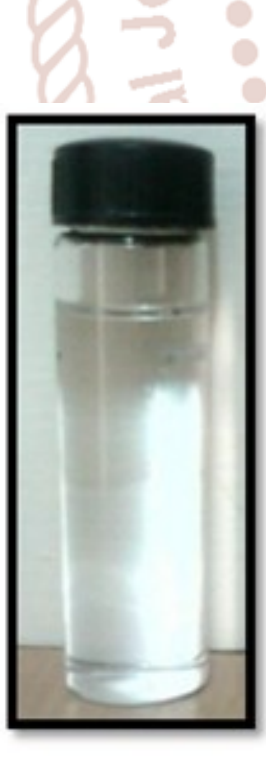

$\mathrm{ZnNO}_{3}$

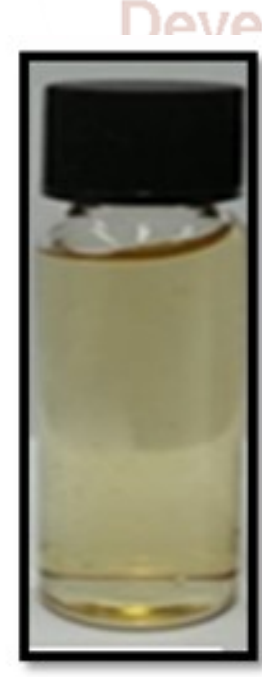

ZnO NPs

Fig. 1: Visual observation of synthesis of Zinc oxide nanoparticles

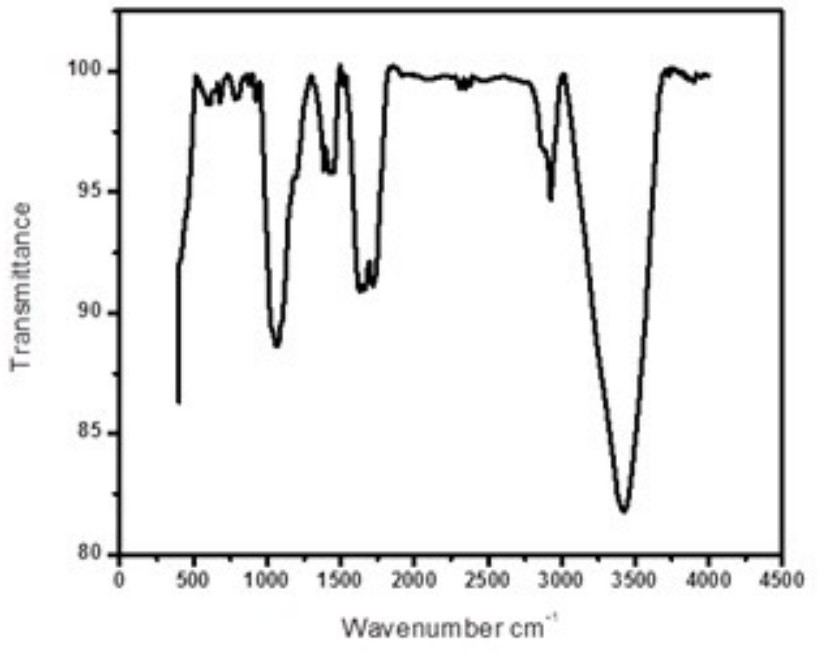

Fig. 3: FTIR spectrum of Zinc oxide nanoparticles

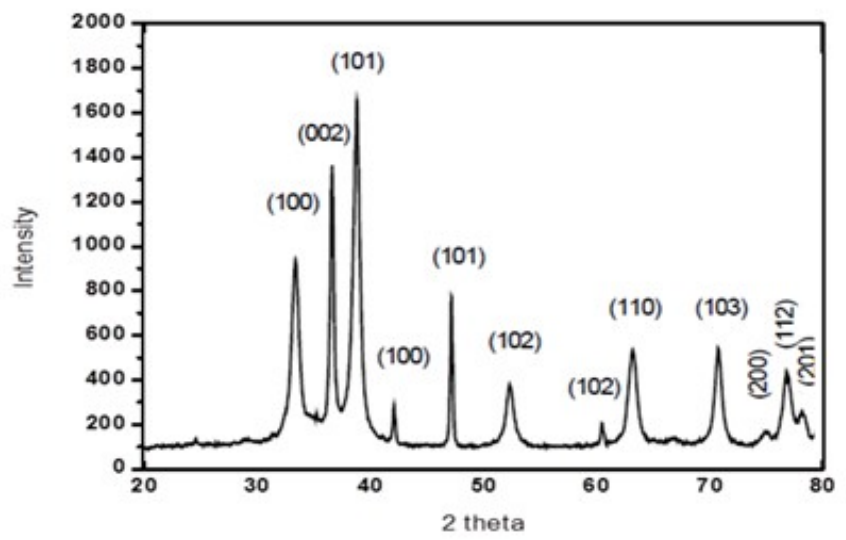

Fig. 4: XRD spectrum of the Zinc oxide nanoparticles 

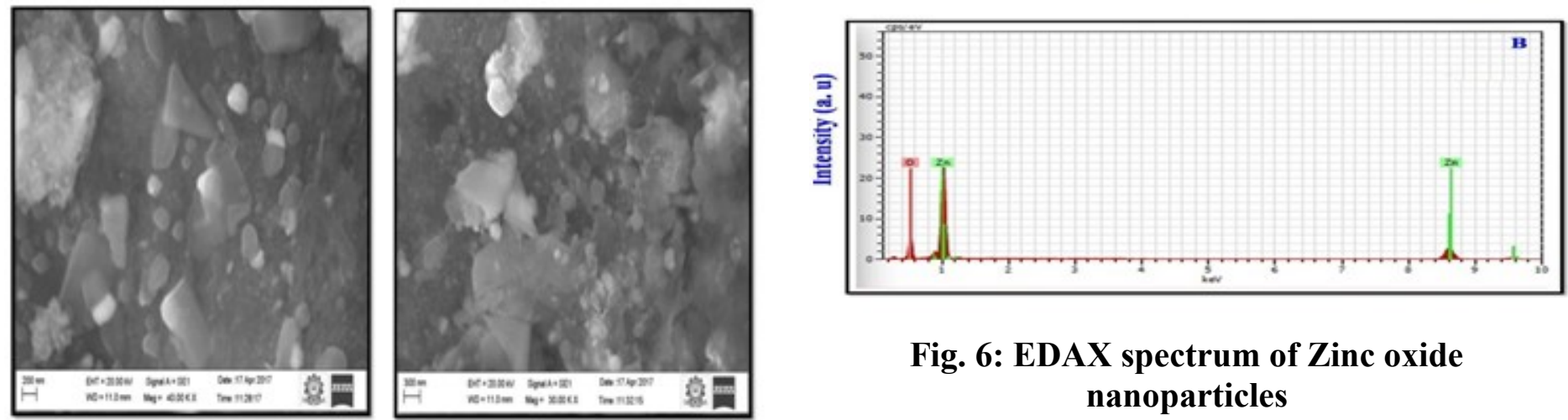

Fig. 6: EDAX spectrum of Zinc oxide nanoparticles
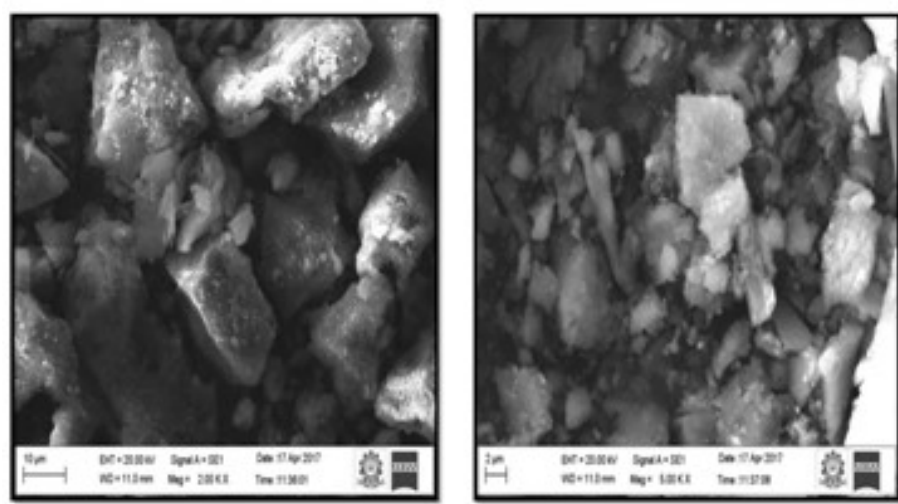

Fig. 5: SEM micrograph of Zinc oxide nanoparticles at different magnifications

Table 1: Antimicrobial activity of Zinc Oxide nanoparticles

\begin{tabular}{|c|c|c|}
\hline \multirow{2}{*}{ Microorganism } & \multicolumn{2}{|c|}{ Diameter of inhibition zone (mm) } \\
\cline { 2 - 3 } & Onion Extract & Zinc oxide \\
nanoparticles
\end{tabular}

